\title{
A MATHEMATICAL MODEL FOR INTERPRETATION OF IMAGES UNDER UNCERTAINTIES IN DIFFERENT FIELDS
}

\author{
Endre Pap ${ }^{1}$, Đorđe Obradović ${ }^{2}$, Zora Konjović ${ }^{2}$ \\ ${ }^{1}$ Singidunum University, Belgrade, Serbia, Obuda University/Institute of Intelligent Engineering Systems, \\ H-1034 Budapest, Hungary \\ ${ }^{1}$ Faculty of Technical Sciences/Department of Computing and Automation, Novi Sad, Serbia
}

\begin{abstract}
:
Real-world objects, in many fields of investigations, are mapped to the digital raster image through a variety of sensors, making the image only an approximation to the real-world object. Due to imperfections in either the image data or the edge detector, there may be missing points or pixels on lines as well as spatial deviations between ideal line and the set of imprecise points obtained from the edge detector. The overall effect is an image that has some distortion in its geometry. It is introduced a mathematical model based on linear fuzzy space, which cover these uncertainties and helps in the correct interpretation and important decisions in different fields: image analysis (imprecise feature extraction), GIS (imprecise spatial object modeling), robotics (environment models), and in medicine (DICOM medical images).
\end{abstract}

\author{
Key words: \\ Image analysis, \\ GIS, \\ DICOM medical images, \\ fuzzy point, \\ linear fuzzy space, \\ fuzzy line, \\ fuzzy triangle.
}

E. P. thanks to the project MNPRS 174009 and project "Mathematical models of intelligent systems and their applications" supported by Provincial Secretariat for Science and Technological Development of Vojvodina.

\section{INTRODUCTION}

The main goal of research topic which is presented in this paper is modeling of basic planar imprecise geometry objects and their relations, as well as their application to spatial data management systems. An overview of the papers dealing with imprecise point objects modeling is given in [1]. In general, three basic approaches to spatial data uncertainty/imprecision are recognized : exact models, probabilistic models and fuzzy models.

Our model of the imprecise line object is based on the model of fuzzy imprecise point presented in [1], as the union of linear combination of two fuzzy points. Fuzzy points are used to model the position of a real object when there is some uncertainty to the measured position. Most often this uncertainty in practical applications is ignored. There are applications in which real objects are not only represented by the position but the entire series of uniformly spaced points. These points can be distributed along a curve that has a beginning and an end. Curve that connects two points is called a line or path. If the points that represent the path are imprecise, then the whole line should be described in way similar to imprecise point's description.

\section{SHORT OVERVIEW OF PREVIOUS METHODS}

We give here only a very short overview of the previously methods used for lane detection systems. the mostly used technique is based on Hough Transform [2], see [3-5]. There are used also many other techniques for lane detection. Pomerleau et al. [6] used neural networks in their ALVINN system. Kang et al. [7] applied connectedcomponent analysis and dynamic programming. Probabilistic methods such as Maximum a posteriori estimation evaluated by Metropolis algorithm used in several systems [8], [9]. Wang et al. [10] applied fuzzy method in feature extraction stage for automatic brightness compensation. Further, lane detection systems represent road lane model as straight lines [11], B-splines [12], parabola [5] and hyperbola [13]. More detailed survey for lane detection strategies can be found on [5]. An overview of the imprecise point objects modeling is given in [1]. Three basic approaches to spatial data uncertainty/imprecision are recognized: exact models [15-18], [12], probabilistic models [8], [9], [19-23] and fuzzy models [1], [24-34]. Löffler [16] has introduced models for imprecise lines as set of possible precise planar lines, while Guibas, Salasin and Stolfi in [14] present basic spatial relations (coincidence, 
between and collinear) between imprecise points modeled as circles with same diameter. Unfortunately, with this approach, it is not possible to describe the line whose points are known with different levels of accuracy. In [28] the problem of spatial objects which do not have homogeneous interiors and sharply defined boundaries is considered. For a spatial vagueness called fuzziness this paper gives a conceptual model of fuzzy spatial objects that also incorporates fuzzy geometric union, intersection, and difference operations as well as fuzzy topological predicates. In [24] the abstract conceptual model of fuzzy spatial data types, such as fuzzy points, fuzzy lines and fuzzy regions, was given. Buckley and Eslami [27] introduced fuzzy plane geometry. The fuzzy point and fuzzy line are defined as fuzzy sets with membership functions that are convex and upper semi-continuous. One of the earliest and the most commonly used technique in feature extraction, known as Generalized Hough transformation, was introduced by Duda and Hart [2]. This technique is based on voting procedure which is carried out in parameter space.

In this paper we will present mathematical model for such fuzzy line. In the sequel, the results belonging to this topic will be briefly presented. We present a new mathematical model of the imprecise basic plane geometric object (fuzzy line, fuzzy triangle), theirs main properties, see [1], [32], see [33]. There was introduced basic distance functions and introduced the basic imprecise spatial relations (coincidence, contain and collinear). Important applications of the introduced mathematical model are obtained in [32] and [34].

\section{LINEAR FUZZY SPACE}

Definition 2.1 Fuzzy point denoted by $\tilde{P}$ is defined by its membership function $\mu_{\tilde{p}}$ from the set which contains all membership functions $u$ satisfying following conditions:

$\left(\forall u \in \mathcal{F}^{2}\right)\left(\exists_{1} P \in \mathbb{R}^{2}\right) u(P)=1$,

$$
\begin{aligned}
& \left(\forall X_{1}, X_{2} \in \mathbb{R}^{2}\right)(\lambda \in[0,1]) u\left(\lambda X_{1}+\right. \\
& \left.(1-\lambda) X_{2}\right) \geq \min \left(u\left(X_{1}\right), u\left(X_{2}\right)\right),
\end{aligned}
$$

function $u$ is upper semi continuous,

$[u]^{\alpha}=\left\{X \mid X \in \mathbb{R}^{2}, u(X) \geq \alpha\right\} \alpha$-cut of function $u$ is convex.

A point $P$ from $\mathbb{R}^{2}$, with membership function $\mu_{\tilde{P}}(P)=1$, will be denoted by $P(P$ is the core of the fuzzy point $\tilde{P}$ ), and the membership function of the point $\tilde{P}$ will be denoted by $\mu_{\tilde{p}}$. By $[P]^{\alpha}$ we denote the $\alpha$-cut of the fuzzy point $\tilde{P}$.

Definition 2.2 Linear fuzzy space $\mathcal{H}^{2}$ is a subset of all functions from Definition 2.1, which in addition to the properties given in Definition 2.1, are:

$$
\begin{aligned}
& (\mu(S)=1), \\
& \mu(V)=\mu(M) \wedge \mu(M) \neq 0 \Rightarrow d(S, V)=d(S, M),
\end{aligned}
$$

where $d(S, M)$ is the distance in $\mathbb{R}^{2}$.

Inverse-linear decreasing with respect to points' distance from the core according to:

$$
\begin{aligned}
& \text { If } r \neq 0 \\
& \mu_{\tilde{S}}(V)=\max \left(0,1-\frac{d(S, V)}{\left|r_{S}\right|}\right), \\
& \text { if } r=0 \\
& \mu_{\tilde{S}}(V)= \begin{cases}1 & \text { if } S=V \\
0 & \text { if } S \neq V,\end{cases}
\end{aligned}
$$

where $d(S, V)$ is the distance between the point $V$ and the core $S\left(V, S \in R^{n}\right)$ and $r \in \mathbb{R}$ is constant.

Elements of that space are represented as ordered pairs $\tilde{S}=\left(S, r_{S}\right)$, where $S \in \mathbb{R}^{2}$ is the core of $\tilde{S}$, and $r_{S} \in \mathbb{R}$ is the distance from the core for which the function value becomes 0 ; in the sequel parameter $r_{S}$ will be denoted as fuzzy support radius.

Definition 2.3 Let the linear fuzzy space be defined on reals. Fuzzy relations $\leq^{R F}$ and $\leq^{L F}$ are defined by membership functions

$$
\begin{gathered}
\left\{\begin{array}{c}
\mu\left(A \leq{ }^{R F} B\right)= \\
0 \quad \text { if } A>B, \\
\frac{B-A}{r_{A}-r_{B}} \text { if } A \leq B \wedge A+r_{A}>B+r_{B} \\
1 \text { if } A \leq B \wedge A+r_{A} \leq B+r_{B},
\end{array}\right. \\
\mu\left(A \leq^{L F} B\right)=\left\{\begin{array}{cl}
0 & \text { if } A>B \\
\frac{B-A}{r_{B}-r_{A}} & \text { if } A \leq B \wedge A-r_{A}>B-r_{B} \\
1 & \text { if } A \leq B \wedge A-r_{A} \leq B-r_{B},
\end{array}\right.
\end{gathered}
$$

respectively, where $\tilde{A}=\left(A, r_{A}\right)$ and $\tilde{B}=\left(B, r_{B}\right)$ are fuzzy points, $A$ is the core of $\tilde{A}$

and $r_{A}$ is a parameter determining the membership function of point $\hat{A}$.

\section{FUZZY PLANE GEOMETRY}

We introduce basic operations over linear fuzzy space $\mathcal{H}^{2}$ defined on $\mathbb{R}^{2}$, as well as their properties which will be used in definitions of basic fuzzy plane geometry objects.

Definition 3.1 Let $\tilde{A}, \tilde{B} \in \mathcal{H}^{2}$. An operator $+: \mathcal{H}^{2} \times \mathcal{H}^{2} \rightarrow \mathcal{H}^{2}$ is called fuzzy points addition given by

$\tilde{A}+\tilde{B}=\left(A+B, r_{A}+r_{B}\right)$,

where $A+B$ is the usual vector addition, and $r_{A}+r_{B}$ is the usual scalar addition.

Definition 3.2 Let $\mathcal{H}^{2}$ be linear fuzzy space. Then a 
function $f: \mathcal{H}^{2} \times \mathcal{H}^{2} \times[0,1] \rightarrow \mathcal{H}^{2}$ is called linear combination of fuzzy points $\tilde{A}, \tilde{B} \in \mathcal{H}^{2}$ is given by

$$
f(\tilde{A}, \tilde{B}, u)=\tilde{A}+u \cdot(\tilde{B}-\tilde{A}),
$$

where $u \in[0,1]$ and operator "." is the scalar multiplication of fuzzy point.

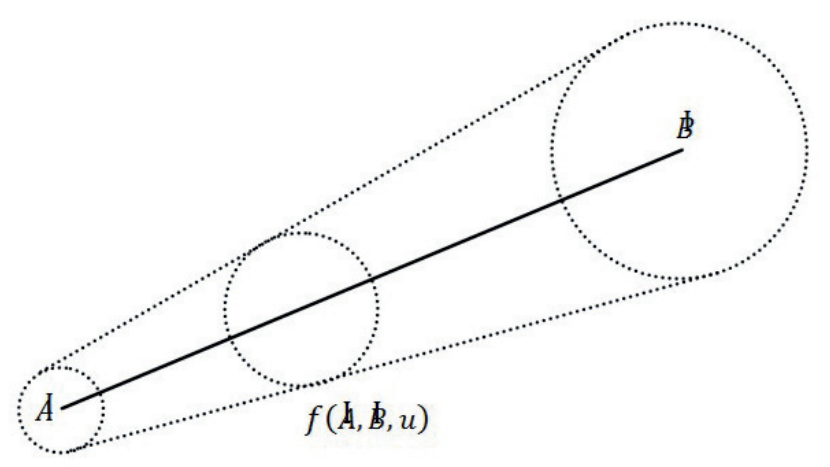

Figure 3.1 Linear combination of the fuzzy points

Definition 3.3 Let $\mathcal{H}^{2}$ be a linear fuzzy space and function $f$ is a linear combination of the fuzzy points $\hat{A}$ and $\tilde{B}$. Then a fuzzy set $\widetilde{A B}$ is called fuzzy line if following holds

$$
\widetilde{A B}=\mathrm{U}_{u \in[0,1]} f(\tilde{A}, \tilde{B}, u) .
$$

Let $\mathcal{H}^{2}$ be linear fuzzy space, fuzzy line $\widetilde{A B}$ defined by fuzzy points $\hat{A}$ and $\tilde{B}$. Then following holds $\widetilde{A B}=\widetilde{B A}$.

\section{FUZZY TRIANGLE}

Definition 4.1 Let $\hat{A}, \tilde{B}, \tilde{C} \in \mathcal{H}^{2}$ be fuzzy points with noncollinear cores $(\tilde{A} \neq \tilde{B} \neq \tilde{C})$ and function $f$ is a linear combination of two fuzzy points. Then the fuzzy set $\overparen{A B C}$ is called fuzzy triangle, if the following holds

$$
\overline{A B C}=\bigcup_{u=0}^{1} f\left(\tilde{A}, \bigcup_{v=0}^{1} f(\hat{B}, C, v), u\right)
$$

Corresponding membership function is denoted by $\mu_{\overparen{A B C}}(X)$ and it is given by to the following formula

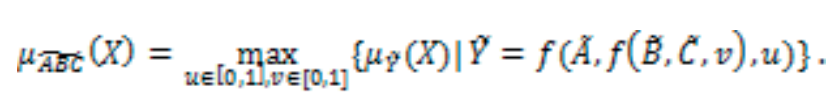

$\alpha_{\text {cut of fuzzy triangle }} \overparen{A B C}$ is denoted by $[\widehat{A B C}]^{\alpha}$.

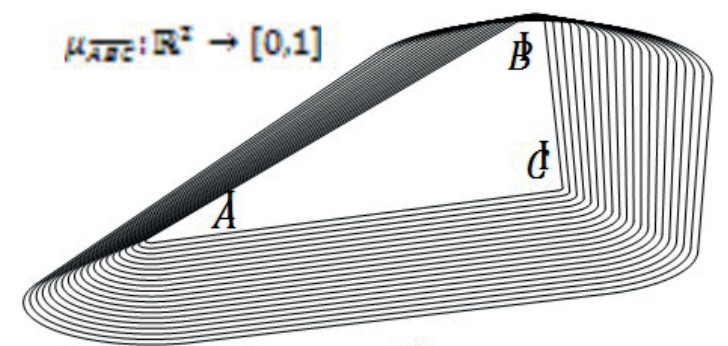

a)

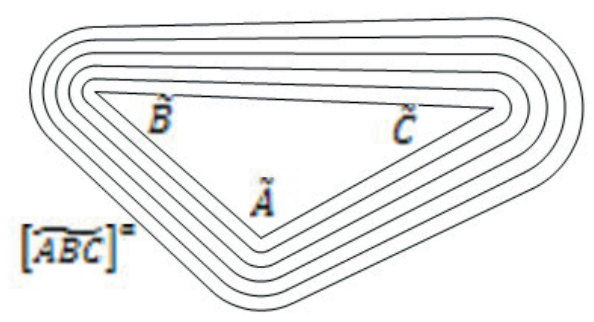

b)

Figure 4.1: a) Fuzzy triangle membership function b) Fuzzy triangle alpha cuts

Definition 4.2 Let $\overparen{A B C}$ be a fuzzy triangle defined on fuzzy linear space $\mathcal{H}^{2}$. Fuzzy point $\tilde{X} \subset \widetilde{A B C}$ is called edge point of the fuzzy triangle $\widetilde{A B C}$ if for all $\alpha \in[0,1]$ a point $Y \in[\tilde{X}]^{\alpha}$ exists such that all its neighborhoods contain at least one point from $[\overparen{A B C}]^{\alpha}$ and at least one point outside of $[\overline{A B C}]^{\alpha}$.

\section{FUZZY SPATIAL RELATIONS}

Measurement of the space especially the distance between plane geometry objects is defined as a generalization of the concept of physical distance. For more general theoretical background for fuzzy approach see [31].

Distance function or metric is a function that behaves according to specific set of rules. There are introduced basic distance functions between fuzzy plane geometry objects and their main properties according to the set of rules presented in our papers [1], [32].

Following distance functions are fuzzy metrics

$$
\begin{aligned}
& d(\tilde{X}, \bar{Y})={ }_{D F}\left(d(X, Y),\left(r_{X}+r_{Y}\right)\right) \\
& d(\tilde{X}, \bar{Y})={ }_{D F}\left(d(X, Y), \max \left(r_{X}, r_{Y}\right)\right) \\
& d(X, Y)={ }_{D F}\left(d(X, Y),\left|r_{X}-r_{Y}\right|\right)
\end{aligned}
$$

Distance (iii) also satisfies set of rulles which hold for the classical metric.

Spatial relations (predicates) are functions that are used to establish mutual relations between the fuzzy geometric objects. The basic spatial relations are coincide, between and collinear. For more details see [32], [33]. Fuzzy relation coincidence expresses the degree of truth that two fuzzy points are on the same place. 
Definition 5.1 Let $\lambda$ be the Lebesgue measure on the unit interval $[0,1]$ and $\mathcal{H}^{2}$ is a linear fuzzy space. The fuzzy relation coin: $\mathcal{H}^{2} \times \mathcal{H}^{2} \rightarrow[0,1]$ is fuzzy coincidence of two fuzzy points $\hat{A}, \widetilde{B}$, represented by the membership function which is the Lebesgue measure of the set of all $\alpha$ for which $\alpha_{\text {cuts of }} \hat{A}$ and $\tilde{B}$ are not disjoint.

Interpretation of the proposition

"Fuzzy point $\hat{A}$ is coincident to fuzzy point $\tilde{B}$ " is partially true with the truth degree

coin: $\mathcal{H}^{2} \times \mathcal{H}^{2} \rightarrow[0,1]$.

In [32], Theorem 4.1, it is given a method for its calculation.

\section{APPLICATIONS}

The proposed model of fuzzy points and imprecise line objects is used in various applications, such as image analysis (imprecise feature extraction), GIS (imprecise spatial object modeling) and robotics (environment models) [1], Figure 6.1.

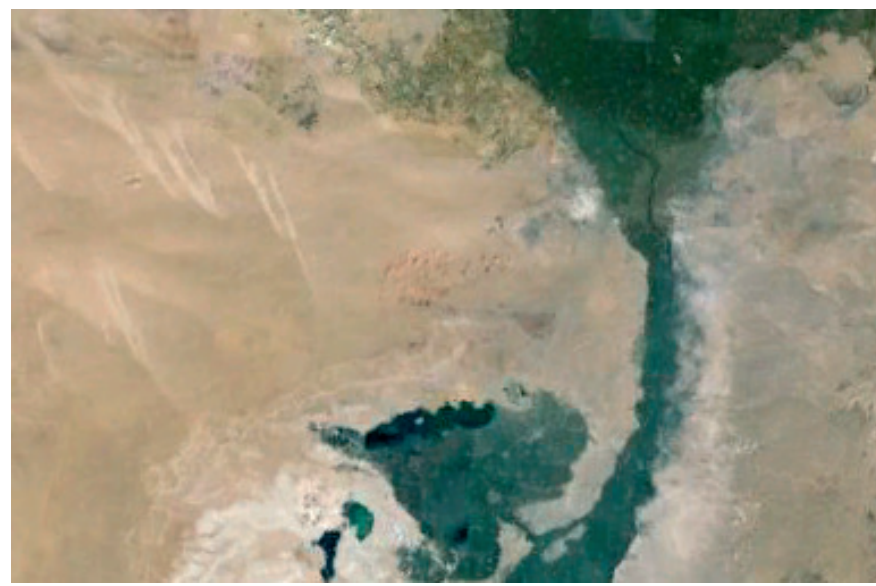

Figure 6.1: Satellite image with a need for a geometric separation between river and earth side

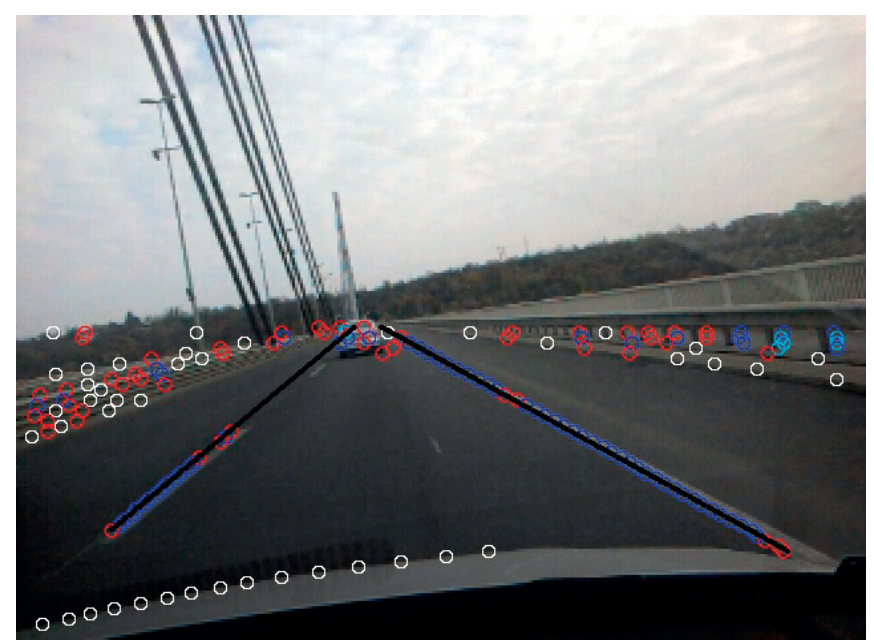

Figure 6.2: Road line (image from the car)
In the our paper [32] we use the notion of the fuzzy line as model of the road lane, Figure 6.2. An algorithm for lane detection is primarily based on fuzzy spatial relations introduced by this model, and it is characterized by reduced computational complexity versus the standard Hough transformation, see also [33].

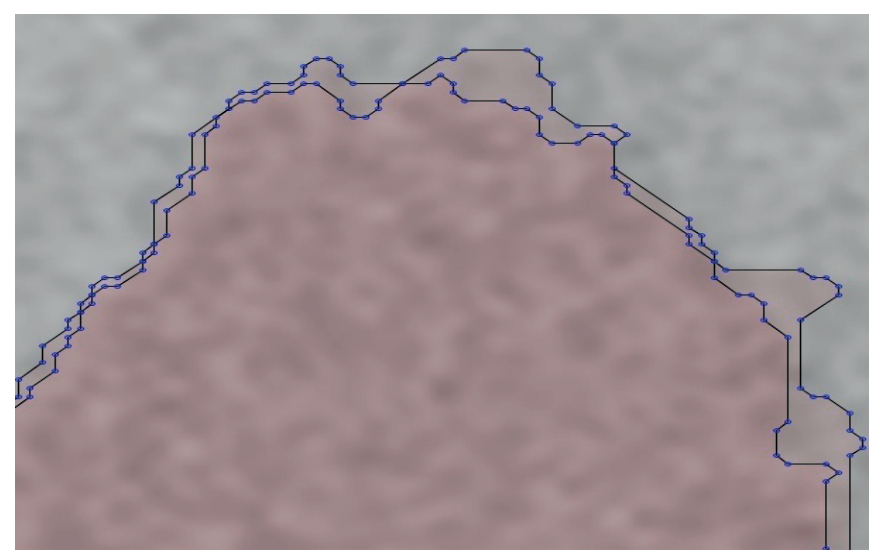

Figure 6.3: Fuzzy line geometry of imprecise regions at DICOM medical image

The constructed model of fuzzy linear space is also used for obtaining fuzzy spatial relations and their interpretations on DICOM medical images, Figures 6.3 and 6.4 , see [34].
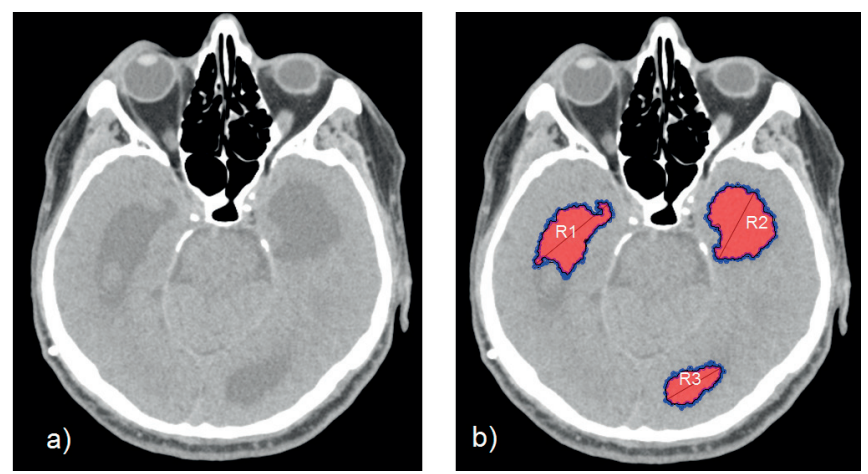

Figure 6.4: a) CT scan , b) Extracted regions

\section{CONCLUSION}

We have briefly presented a mathematical model based on the notion of fuzzy linear space, and three real applications. Our final goal is to develop an effective applicable framework for dealing with imprecise spatial data. For that purpose it is necessary to develop new specialized fuzzy indexing structures analogous to $\mathrm{R}$ tree, Quad tree and GRID. Namely, this is one of the main research directions related to the development of fuzzy linear space-based algorithms. Another future research direction would be in the extension of the introduced two dimensional concepts to the three dimensional linear fuzzy space. 


\section{REFERENCES}

[1] Đ. Obradović, Z. Konjović, E. Pap, and N. M. Ralević, “The maximal distance between imprecise point objects," Fuzzy Sets and Systems, vol. 170, no. 1, 2011, pp. 76-94.

[2] R. O. Duda and P. E. Hart, "Use of the Hough transformation to detect lines and curves in pictures," Communications of the ACM, vol. 15, no. 1, Jan. 1972, pp. 11-15.

[3] Qing Li, Nanning Zheng, and Hong Cheng, "Springrobot: a prototype autonomous vehicle and its algorithms for lane detection," IEEE Transactions on Intelligent Transportation Systems, vol. 5, no. 4, Dec. 2004,pp. 300- 308.

[4] B. Yu and A. K. Jain, "Lane boundary detection using a multiresolution Hough transform," in , International Conference on Image Processing, 1997. Proceedings, 1997, vol. 2, pp. $748-751$.

[5] J. C. McCall and M. M. Trivedi, "Video-Based Lane Estimation and Tracking for Driver Assistance: Survey, System, and Evaluation," IEEE Transactions on Intelligent Transportation Systems, vol. 7, Mar. 2006, pp. 20-37.

[6] D. A. Pomerleau, "Neural Network Vision for Robot Driving," THE HANDBOOK OF BRAIN THEORY AND NEURAL NETWORKS, 1996, pp. 161-181.

[7] D.-J. Kang and M.-H. Jung, "Road lane segmentation using dynamic programming for active safety vehicles," Pattern Recogn. Lett., vol. 24, no. 16, Dec. 2003, pp. 3177-3185.

[8] C. Kreucher, S. Lakshmanan, and K. Kluge, "A driver warning system based on the LOIS LANE detection algorithm," in Proceedings of the 1998 IEEE International Conference on Intelligent Vehicles, 1998.

[9] C. Kreucher and S. Lakshmanan, "LANA: a lane extraction algorithm that uses frequency domainfeatures," IEEE Transactions on Robotics and Automation, vol. 15, no. 2, Apr. 1999, pp. 343-350.

[10] J.-G. Wang, C.-J. Lin, and S.-M. Chen, “Applying fuzzy method to vision-based lane detection and departure warning system," Expert Syst. Appl., vol. 37, no. 1, Jan. 2010, pp. 113-126.

[11] K. Chen and W. Tsai, "Vision based autonomous land vehicle guidance in outdoor road environments using combined line and road following techniques," Journal of Robotic Systems, vol. 14, no. 10, Oct. 1997, pp. 711-728.

[12] Yue Wang, Eam Khwang Teoh, and Dinggang Shen, "Lane detection using B-snake,” pp. 438-443.

[13] L. Bai, Y. Wang, and M. Fairhurst, “An extended hyperbola model for road tracking for video-based personal navigation," Know.-Based Syst., vol. 21, no. 3, Apr. 2008, pp. 265272.

[14] L. Guibas, D. Salesin, and J. Stolfi, "Epsilon geometry: building robust algorithms from imprecise computations," in Proceedings of the fifth annual symposium on Computational geometry, New York, NY, USA, 1989, pp.208-217.

[15] M. Löffler and M. van Kreveld, "Geometry with Imprecise Lines," in 24th European Workshop on Computational Geometry, 2008.

[16] M. Löffler, Data Imprecision in Computational Geometry, PhD Thesis. Utrecht: Utrecht University, 2009.
[17] M. Löffler and M. Kreveld, "Largest and Smallest Convex Hulls for Imprecise Points," Algorithmica, vol. 56, no. 2 ,2008, pp.235-269.

[18] K. Yang, S. Sam Ge, and H. He, "Robust line detection using two-orthogonal direction image scanning," Computer Vision and Image Understanding, vol. 115, no. 8, 2011, pp. 1207-1222.

[19] N. Kiryati, Y. Eldar, and A. M. Bruckstein, “A probabilistic Hough transform," Pattern Recognition, vol. 24, no. 4, 1991, pp. 303-316.

[20] R. S. Stephens, "Probabilistic approach to the Hough transform," Image and vision computing, vol. 9, no. 1, 1991, pp. $66-71$.

[21] C. Galambos, J. Matas, and J. Kittler, "Progressive probabilistic Hough transform for line detection," in Computer Vision and Pattern Recognition, 1999. IEEE Computer Society Conference on., 1999, vol. 1.

[22] J. Matas, C. Galambos, and J. Kittler, "Robust Detection of Lines Using the Progressive Probabilistic Hough Transform," Computer Vision and Image Understanding, vol. 78, no. 1, 2000, pp. 119-137.

[23] C. Galambos, J. Kittler, and J. Matas, "Using gradient information to enhance the progressive probabilistic Hough transform," in Proceedings 15th International Conference on Pattern Recognition. ICPR-2000, Barcelona, Spain, pp. 560-563.

[24] M. Schneider, "Fuzzy topological predicates, their properties, and their integration into query languages," in Proceedings of the 9th ACM international symposium on Advances in geographic information systems, 2001, pp. 9-14.

[25] A. Rosenfeld, “The fuzzy geometry of image subsets," Pattern Recognition Letters, vol. 2, no. 5, 1984, pp. 311-317.

[26] M. Schneider, "Design and implementation of finite resolution crisp and fuzzy spatial objects," Data Knowl. Eng., vol. 44, 2003, pp. 81-108.

[27] J. J. Buckley and E. Eslami, "Fuzzy plane geometry I: Points and lines," Fuzzy Sets and Systems, vol. 86, no. 2,1997, pp. 179-187.

[28] M. Schneider, Spatial Data Types for Database Systems: Finite Resolution Geometry for Geographic Information Systems, 1st ed. Springer, 1997.

[29] Đ. Obradović, Z. Konjović, and E. Pap, "Extending PostGIS by imprecise point objects," in IEEE 8th International Symposium on Intelligent Systems and Informatics, Subotica, Serbia, 2010, pp. 23-28.

[30] J. Verstraete, G. Tré, R. Caluwe, and A. Hallez, "Field based methods for the modeling of fuzzy spatial data," Fuzzy Modeling with Spatial Information for Geographic Problems, 2005, pp. 41-69.

[31] O. Hadžić and E. Pap, Fixed point theory in probabilistic metric spaces. Dordecht: Kluwer Academic Publishers, 2001.

[32] Đ. Obradović, Z. Konjović, E. Pap, and I. J. Rudas, "Linear Fuzzy Space Based Road Lane Model and Detection," Knowledge-Based Systems 38, 2013, pp. 37-48. 
[33] Đ. Obradović, Z. Konjović, E. Pap, and I. J. Rudas,” Fuzzy geometry in linear fuzzy space," in Intelligent Sysstems: Models and Applications, E. Pap, Ed., Springer, 2013, pp. 137-153.

[34] M. Jocić, Đ. Obradović, Z. Konjović and E. Pap, “Fuzzy spatial relations and their applications on DICOM medical images, IEEE $11^{\text {th }}$ International Symposium on Intelligent Systems and Informatics (SISY 2013), Subotica, 39-44. 